\title{
Photometric variability in the open cluster M 67
}

\section{Cluster members detected in X-rays}

\author{
M. van den Berg ${ }^{1, \star}$, K. G. Stassun ${ }^{2}$, F. Verbunt ${ }^{1}$, and R. D. Mathieu ${ }^{2}$ \\ 1 Astronomical Institute, Utrecht University, PO Box 80000, 3508 TA Utrecht, The Netherlands \\ 2 Department of Astronomy, University of Wisconsin-Madison, 475 N Charter St, Madison, WI 53706, USA
}

Received 15 January 2001/ Accepted 23 October 2001

\begin{abstract}
We study photometric variability among the optical counterparts of X-ray sources in the old open cluster M67. The two puzzling binaries below the giant branch are both variables: for S1113 the photometric period is compatible with the orbital period, S 1063 either varies on a period longer than the orbital period, or does not vary periodically. For the spectroscopic binaries S 999, S 1070 and S 1077 the photometric and orbital periods are similar. Another new periodic variable is the main-sequence star S1112, not known to be a binary. An increase of the photometric period in the W UMa system S 1282 (AH Cnc) is in agreement with a previously reported trend. Six of the eight variables we detected are binaries with orbital periods of 10 days or less and equal photometric and orbital periods. This confirms the interpretation that their X-ray emission arises in the coronae of tidally locked magnetically active stars. No variability was found for the binaries with orbital periods longer than 40 days; their X-ray emission remains to be explained.
\end{abstract}

Key words. stars: activity - binaries: general - stars: variables: general - open clusters and associations: individual: M 67 - X-rays: stars

\section{Introduction}

Twenty five members of the old open cluster M67 have been detected in X-rays (Belloni et al. 1998). At the age of M 67 (4 Gyr, Pols et al. 1998) the rotation of single stars is too slow to generate detectable X-rays. Therefore, the X-ray emission of many M67 sources probably arises in interacting binaries. Indeed, one source is known to be a cataclysmic variable. Nine sources are binaries with orbital periods of 10 days or less, presumably RS CVn type systems, whose X-rays are due to the coronae of magnetically active stars forced to corotate by tidal interaction (see Table 2). However, not all X-ray sources are binaries, e.g. one source is a hot white dwarf, and some others are stars which do not show signs of binarity.

There are five peculiar binaries (S 1040, S 1063, S 1072, S 1082 and S 1113) whose evolutionary statuses we currently do not understand. They are found in the colourmagnitude diagram in locations which cannot be reproduced by combining the light from any two members on the main sequence, subgiant and/or giant branches. Two of them have long orbital periods which exclude strong

Send offprint requests to: M. van den Berg,

e-mail: vdberg@merate.mi.astro.it

* Present address: Osservatorio Astronomico di Brera, Via E. Bianchi 46, 23807 Merate (LC), Italy tidal interaction. A sixth binary (S 1237) with a long orbital period lies to the blue of the giant branch which can be explained by the superposition of the light of a giant and a turnoff star.

A spectroscopic study of these peculiar systems was presented in van den Berg et al. (1999); see their Fig. 1 for the position of these systems in the colour-magnitude diagram of M 67. Here we report our photometric study of these sources and of other X-ray sources that happen to be in the same fields of view. The photometry of S 1082 is published separately (van den Berg et al. 2001). The observations and analyses are described in Sect. 2. Results are presented in Sect. 3, followed by the interpretation and discussion - including comparison with earlier work - in Sect. 4. Section 5 summarizes our conclusions. The variability of stars not detected in X-rays but included in our observations will be the subject of Paper II (Stassun et al. 2002).

\section{Data and analysis}

\subsection{Observations}

$U, B, V, I$ and Gunn $i$ photometry was obtained with the $0.90 \mathrm{~m}$ telescope at Kitt Peak, the $0.91 \mathrm{~m}$ ESO-Dutch Telescope at La Silla and the $1 \mathrm{~m}$ Jacobus Kapteyn 
Telescope on La Palma. Combined, the five observation runs span a period of two years (see Table 1). Figure 1 shows the location of the observed fields. During the first run weather conditions were good with a typical seeing of 0.9 to $1^{\prime \prime} .4$. The observations were made during and around full moon but moon illumination was not a problem. Weather conditions during runs 2 and 3 were good, with a typical seeing of 1 .' 6 . During run 4 the typical seeing was 1 ." 5 while the quality of some images was affected by the brightness of the nearby moon. The same, at a seeing between $1^{\prime \prime} 5$ and $3^{\prime \prime}$, is true for the last run, that in addition was troubled by partial cloudiness. This is reflected in the relatively large errors of the last two runs.

Every X-ray source of Belloni et al. (1998) was monitored in at least one run, except for the faint cataclysmic variable EU Cnc and the hot white dwarf. The main purpose of run 1 was to monitor variability of $\mathrm{S} 1113$ and $\mathrm{S} 1063$, of run 2 to monitor S 1113 and of runs 3 to 5 to monitor S 1082. This means that exposure times were chosen to optimize the measurements of these stars. During run 5 , five additional fields containing X-ray sources were observed in $B$ and $V$ once or twice per night to search for obvious signs of variability. As S 364 and S 1237 are very bright stars (see Table 2) the quality of the images of fainter stars in these fields is poor. This affects the quality of the light curves of the X-ray sources in the field of view of S 1237 (i.e. S 1242, S 1270 and S 1282).

\subsection{Data reduction and light curve solution}

Standard IRAF $^{1}$ routines were used to remove the bias signal and flatfield the images. Aperture photometry for all the stars was done with the DAOРнот.Pнот task. For each individual run, source counts were extracted within a fixed radius with a value depending on the seeing conditions. The stars in M67 are separated well enough to avoid problems of crowding.

The light curve solution was computed with the algorithm of ensemble photometry as described by Honeycutt (1992). In this method, the magnitudes of all the stars on every frame are used to create an ensemble average with respect to which the brightness variations are defined. Frames that have a large offset from this average (e.g. due to bad seeing) show up as deviant observations and can be excluded. For a given star, errors were assigned to the data points by estimating the typical spread in the light curves of stars of similar magnitude. In most cases the formal errors from the PHOT task are negligible; if not, we used this error instead. The different datasets were analyzed individually.

As exposure times were chosen to optimize the measurements of the X-ray sources in each field, the photometric precision as a function of stellar brightness varies from

\footnotetext{
${ }^{1}$ IRAF is distributed by the National Optical Astronomy Observatories, which are operated by the Association of Universities for Research in Astronomy, Inc., under cooperative agreement with the National Science Foundation.
}

one run to the next. Generally speaking, the photometric precision of the brightest (unsaturated) stars in our exposures is flat-field limited to $5-10$ mmag. This precision level typically holds for stars up to $2-2.5$ mag fainter than the brightest sources, and then becomes photon-noise limited and degrades for still fainter stars. The best overall precision was achieved on our Kitt Peak frames (run 1; Table 1$)$, for which the brightest stars $(B \approx 12, V \approx 12$, $I \approx 11.5$ ) have $\sigma_{\mathrm{mag}}=0.007,0.005,0.005$ in $B, V$, and $I$, respectively. The precision begins to degrade at around 14 th mag. For the faintest sources, at about $18.5 \mathrm{mag}$, the precision is $0.05-0.1 \mathrm{mag}$. We refer the reader to Paper II for a full discussion of the photometric precision in our observations.

A simple zero-point shift is applied to the measurements in each filter to roughly place the instrumental magnitudes on an absolute scale as described in Paper II. The light and colour curves that are presented in Figs. 3-5 show the variations with respect to the mean magnitude and colour as listed in Table 3 of Paper II.

\subsection{Search for variability}

Our search for variability is a two-step process. First we perform a $\chi^{2}$-test on the individual light curves for each filter for each run, to calculate the probability that the light curves of the X-ray sources are compatible with being constant. As the intrinsic properties of the variability need not be the same in light curves of different runs (in particular for brightness variations due to spots), we consider each light curve separately. To remove accidental outliers, the minimum and maximum data points are excluded. A star is listed as a variable in column "var" of Table 2 if the $\chi^{2}$-test predicts for any of its light curves that the probability for it being constant is smaller than $0.3 \%$. Eight stars are thus marked as probable variables. For six of these stars the variability is not detected in every light curve. In most cases we can ascribe this to differences in sensitivity between runs or between different filters of a certain run, or to different durations of runs. For S 1063, S 1070 and S 1077 it seems that the variability itself has changed as discussed below.

We next perform a Lomb-Scargle time-series analysis (Scargle 1982) to search for periodicity in the light curves. In cases when multiple light curves in a given filter are marked as variable, those light curves are combined for the period search; no distinction was made between $I$ and Gunn $i$. If the resulting period does not produce a smooth folded light curve, data from different runs are analyzed separately; this will be indicated for each source in Sect. 3. A periodogram is computed with 1000 frequencies between a minimum and maximum period $P_{\min }$ and $P_{\max }$ corresponding to twice the typical sampling period and the full length of the longest observation run, respectively (see Table 1). We choose the period of the highest peak in the periodogram as our first estimate for periodicity in the data. However, as will be discussed below external 
Table 1. Log of the observations. From left to right: number and dates of the observation run; telescope; Sanders number of the star near the centre of the field; field of view; filters; typical exposure time in seconds for each filter; the minimum and maximum period $P_{\min }$ and $P_{\max }$ used to compute periodograms for the specified run.

\begin{tabular}{|c|c|c|c|c|c|c|c|c|}
\hline Run & Dates & Telescope & Centre & FOV & Filters & $t_{\exp }(\mathrm{s})$ & $P_{\min }$ & $P_{\max }$ \\
\hline 1 & Jan. 4-16 1998 & 0.90m KPNO & S 1084 & $23^{\prime} \times 23^{\prime}$ & $B V I$ & 906060 & $2.4 \mathrm{hr}$ & $10 \mathrm{~d}$ \\
\hline 2 & $\begin{array}{l}\text { Feb. 12, } 14-17,21,24-28 \\
\text { 1998, Mar. 1-3, 6, } 81998\end{array}$ & 0.91m ESO Dutch & S 1113 & $3.8 \times 3.8$ & $U B V$ Gunn $i$ & 300120120120 & $5 \mathrm{hr}$ & $25 \mathrm{~d}$ \\
\hline 3 & Feb. 2-19 1999 & 0.91m ESO Dutch & S 1068* & $3.8 \times 3.8$ & $U B V$ Gunn $i$ & 3601005030 & $30 \mathrm{mn}$ & $18 \mathrm{~d}$ \\
\hline 4 & Dec. 25, 261999 & 1m ING JKT & S 1082 & $10^{\prime} \times 10^{\prime}$ & $B V$ & 7530 & $12 \mathrm{mn}$ & $1 \mathrm{~d}$ \\
\hline \multirow[t]{6}{*}{5} & Feb. $13-16,202000$ & 1m ING JKT & S 1082 & $10^{\prime} \times 10^{\prime}$ & $U B V I$ & 35030158 & $30 \mathrm{mn}$ & $8 \mathrm{~d}$ \\
\hline & & & S 364 & $10^{\prime} \times 10^{\prime}$ & $B V$ & 154 & $4 \mathrm{hr}$ & $8 \mathrm{~d}$ \\
\hline & & & S 628 & $10^{\prime} \times 10^{\prime}$ & $B V$ & 15080 & $4 \mathrm{hr}$ & $8 \mathrm{~d}$ \\
\hline & & & S 1013 & $10^{\prime} \times 10^{\prime}$ & $B V$ & 208 & $4 \mathrm{hr}$ & $8 \mathrm{~d}$ \\
\hline & & & S 1113 & $10^{\prime} \times 10^{\prime}$ & $B V$ & 200100 & $4 \mathrm{hr}$ & $8 \mathrm{~d}$ \\
\hline & & & S 1237 & $10^{\prime} \times 10^{\prime}$ & $B V$ & 2010 & $4 \mathrm{hr}$ & $8 \mathrm{~d}$ \\
\hline
\end{tabular}

* 1063 starting from February 8.

information often leads us to immediately neighbouring peaks of comparable significance.

Photometric periods have been determined previously for the two contact binaries S 1036 (Gilliland et al. 1991) and S 1282 (e.g. Kurochkin 1979). Therefore, we look for periods in a narrow window instead of the range limited by $P_{\min }$ and $P_{\max }$. In both cases, we find that the power at half the photometric period is far higher than at the photometric period, due to the symmetry in the light curve. For $\mathrm{S} 1036$ we search for periods between 0.215 and 0.225 days, for $\mathrm{S} 1282$ between 0.175 and 0.185 days. The periodogram is computed for 5000 points to increase the resolution.

An estimate for the chance detection of a period, i.e. the probability that the light curve does not have the periodicity indicated by the highest peak, is expressed by the false alarm probability. In the case of the Lomb-Scargle periodogram the false alarm probability follows the expression: $1-[1-\exp (-z)]^{m}$, where $z$ is the height of the peak and $m$ is the number of independent frequencies. Horne \& Baliunas (1986) demonstrated that this number can be smaller than the number of data points especially in sets of unevenly sampled data. The value of $m$ is obtained by fitting this expression to a probability distribution generated by measuring the maximum peak heights in periodograms of 5000 simulated random datasets with the same time-sampling and the same spread in the measurements as the actual light curve.

Photometric periods with a false alarm probability smaller than $1 \%$ are summarized in Table 2. These correspond to the position of the highest peak in the periodogram unless indicated otherwise. To estimate the error in the best period we proceed as follows. For the correct period the light curves defined by the first and last observations coincide. A small change $\mathrm{d} P$ in the period causes a small phase shift: $\mathrm{d} \phi=T /(P+\mathrm{d} P)-T / P$, where $T$ is the timespan of the dataset. For each light curve we estimate, by visual inspection, a maximum $\mathrm{d} \phi_{\max }$ for which the light curves do not split perceptibly. This corresponds to a maximum acceptable period change of

$\frac{\mathrm{d} P}{P}=\frac{-\mathrm{d} \phi_{\max } P}{T+\mathrm{d} \phi_{\max } P}$.

The value for $\mathrm{d} \phi_{\max }$ that we choose is listed in Table 2 .

\section{Results}

We have divided the sources into periodically (Sect. 3.1) or non-periodically (Sect. 3.2) varying stars. The periodically varying stars are two W UMa systems, four spectroscopic binaries and one star not known to be a binary, i.e. S 1112.

\subsection{Periodic variables}

\subsubsection{W UMa systems}

The W UMa light curves are plotted versus photometric phase where phase 0 corresponds to the moment of photometric primary minimum.

$\mathbf{S} \mathbf{1 2 8 2}$ is the W UMa variable AHCnc discovered by Kurochkin (1960). The periodograms of the total $B$ and $V$ datasets show peaks with a spacing of $\sim 4 \times 10^{-5}$ and $\sim 6 \times 10^{-4}$ days due to the two-year and the two-month gaps in our observations, respectively (see Fig. 2). The highest peaks in the $B$ and $V$ periodograms are found at 0.180226 and 0.180270 days, respectively, which corresponds to two neighbouring peaks in the periodogram. We folded the data on twice those periods but find that the peak at 0.180226 represents best the true period: when we use the longer period, the deeper primary minima of the first run fall on top of the secondary minima of the fourth run. Thus we conclude that the photometric period is 0.360452 days. $I$ data were only obtained during run 1 and cannot provide an equally precise period.

In addition to short-term variations on a time scale of roughly 9 to 10 years, Kurochkin (1979) finds a secular 


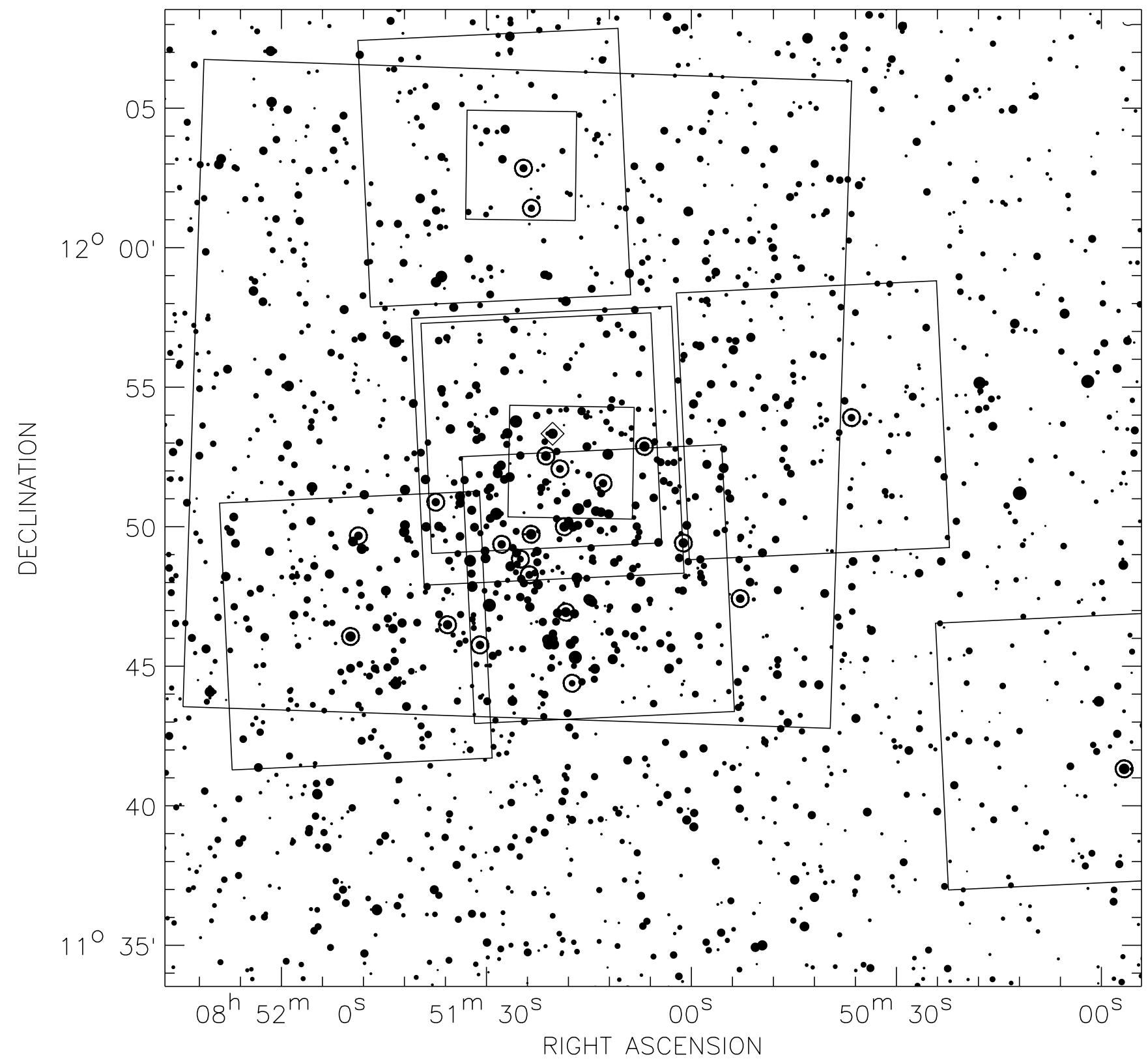

Fig. 1. A $35^{\prime} \times 35^{\prime}$ region of M 67 centred on the star S 783. The coordinates of the sources in this field are taken from the USNO-A1.0 catalogue (epoch J2000). The areas monitored in the five observation runs are indicated by the squares (see also Table 1). The open circles mark the optical counterparts to the X-ray sources that are discussed in this paper; the diamond marks S 1082 that we discuss elsewhere (van den Berg et al. 2001).

increase of the period of AH Cnc. His ephemeris for the primary minimum is:

$$
\begin{aligned}
\operatorname{Min} I= & 2441740.7166(27)+0 \mathrm{~d} 36044098(53) E \\
& +1.56(38) \times 10^{-10} E^{2} .
\end{aligned}
$$

This ephemeris predicts a period during the time of our observations between 0 d 3604447 and 0.3604479 , in agreement with our result. We note that period changes of similar magnitude have been found for other contact binaries. $B-V$.
S 1036, or EV Cnc, was discovered to be a contact binary by Gilliland et al. (1991) who report a period of 0.44125 days. The $B$ and $V$ periodograms show fine structure from the two-year and two-month gaps in the data. In both sets, that include data from run 1, 4 and 5, we find the same best period of 0.22078 days. When folded on this period, the light curves show the same effect of interchanging primary and secondary minima as described for S1282. Proper phasing is obtained with the period of 0.22072 days derived from the peak next to the highest. The period that corresponds to the highest peak in the periodogram of the $I$ data is 0.22091 days, but again 
Table 2. Properties of the X-ray sources of Belloni et al. (1998) discussed in this paper. From left to right: Sanders number (Sanders 1977); X-ray source number from Belloni et al. (1998); $V$ magnitude and $B-V$ colour (Montgomery et al. 1993); for spectroscopic binaries: orbital period $P_{\text {orb }}$ in days and eccentricity $e$; observation runs during which the star was observed; a variability indicator $n(y)$ if the probability that the source is constant is larger (smaller) than $0.3 \%$; photometric period $P_{\text {phot }}$ in days with the run number(s) and the filter for which the period was detected in square brackets; maximum phase shift used to estimate the error in the period; logarithm of the false alarm probability of the period detection; remarks and references for information on the spectroscopic binaries ( 1 = Mathieu et al. 2002, in preparation, 2 = Mathieu et al. $(1990), 3=$ preliminary solution by Latham et al., private communication).

\begin{tabular}{|c|c|c|c|c|c|c|c|c|c|c|c|c|}
\hline$S$ & $X$ & $V$ & $B-V$ & $P_{\text {orb }}(\mathrm{d})$ & $e$ & run & var & & ot $(\mathrm{d})$ & $\mathrm{d} \phi_{\max }$ & $\log$ FAP & remarks \\
\hline 364 & 19 & 9.84 & 1.36 & & & 5 & $\mathrm{n}$ & & & & & \\
\hline 628 & 35 & 14.47 & 0.76 & & & 5 & $\mathrm{n}$ & & & & & \\
\hline 760 & 49 & 13.29 & 0.57 & $954(12)$ & $0.43(9)$ & 1 & $\mathrm{n}$ & & & & & 3 \\
\hline 775 & 44 & 12.69 & 0.63 & & & 1,5 & $\mathrm{n}$ & & & & & \\
\hline 972 & 17 & 15.37 & 0.89 & $1.166412(2)$ & $0.009(6)$ & 1,5 & $\mathrm{n}$ & & & & & 3 \\
\hline 999 & 13 & 12.60 & 0.78 & $10.05525(19)$ & 0 & 1,5 & $\mathrm{y}$ & $9.2(2.0)^{a}$ & {$[1 ; B]$} & 0.3 & -2.62 & 2 \\
\hline 1019 & 11 & 14.34 & 0.81 & $1.360217(18)$ & $0.023(13)$ & 1,5 & $\mathrm{n}$ & & & & & 3 \\
\hline 1027 & 46 & 13.24 & 0.60 & & & $1,4,5$ & $\mathrm{n}$ & & & & & \\
\hline 1036 & 45 & 12.78 & 0.49 & & & $1,4,5$ & $\mathrm{y}$ & $\begin{array}{l}0.44144(1)^{a} \\
0.44144(1)^{a} \\
0.44144(1)^{a}\end{array}$ & $\begin{array}{l}{[\text { all; } B]} \\
{[\text { all; } V]} \\
{[1+5 ; I]}\end{array}$ & $\begin{array}{l}0.05 \\
0.05 \\
0.05\end{array}$ & $\begin{array}{l}-18.6 \\
-17.0 \\
-6.4\end{array}$ & EV Cnc \\
\hline 1040 & 10 & 11.52 & 0.87 & $42.8271(22)$ & 0. & $1,4,5$ & $\mathrm{n}$ & & & & & \\
\hline 1045 & 41 & 12.54 & 0.59 & 7.64521(11) & 0. & $1,4,5$ & $\mathrm{n}$ & & & & & \\
\hline 1063 & 8 & 13.79 & 1.05 & $18.396(5)$ & $0.206(14)$ & $1,3,4,5$ & $\mathrm{y}$ & $\left(17-18^{b}\right)$ & $\begin{array}{l}{[1 ; U],} \\
{[1+3 ; B, V, I]}\end{array}$ & & & 1 \\
\hline 1070 & 38 & 13.90 & 0.63 & $2.66059(8)$ & 0. & $1,3,4,5$ & $\mathrm{y}$ & $\begin{array}{l}2.6(1) \\
2.6(1)\end{array}$ & $\begin{array}{l}{[3 ; B]} \\
{[3 ; V]}\end{array}$ & $\begin{array}{l}0.3 \\
0.3\end{array}$ & $\begin{array}{l}-17.6 \\
-31.5\end{array}$ & 3 \\
\hline 1072 & 37 & 11.32 & 0.61 & 1495(16) & $0.32(7)$ & $1,3,4,5$ & $\mathrm{n}$ & & & & & 2 \\
\hline $1077^{c}$ & 7 & 12.60 & 0.64 & $1.358766(8)$ & $0.095(33)$ & $1,3,5$ & $\mathrm{y}$ & $\begin{array}{l}1.42(9) \\
1.44(9)^{a} \\
1.38(9)\end{array}$ & $\begin{array}{l}{[3 ; B]} \\
{[3 ; V]} \\
{[3 ; I]}\end{array}$ & $\begin{array}{l}0.3 \\
0.3 \\
0.3\end{array}$ & $\begin{array}{l}-3.3 \\
-7.2 \\
-5.5\end{array}$ & 3 \\
\hline 1112 & 28 & 14.98 & 0.78 & & & $1,2,5$ & $\mathrm{y}$ & $\begin{array}{l}2.7(2) \\
2.65(3)\end{array}$ & $\begin{array}{l}{[1 ; B]} \\
{[1+2 ; V]}\end{array}$ & $\begin{array}{l}0.3 \\
0.3\end{array}$ & $\begin{array}{l}-3.0 \\
-7.8\end{array}$ & \\
\hline 1113 & 26 & 13.77 & 1.01 & $2.823105(14)$ & $0.022(10)$ & $1,2,5$ & $\mathrm{y}$ & $\begin{array}{l}2.84(8) \\
2.834(1) \\
2.833(1) \\
2.84(3)\end{array}$ & $\begin{array}{l}{[2 ; U]} \\
{[\text { all; } B]} \\
{[\text { all; } V]} \\
{[1+2 ; I]}\end{array}$ & $\begin{array}{l}0.1 \\
0.1 \\
0.1 \\
0.1\end{array}$ & $\begin{array}{l}-3.9 \\
-14.5 \\
-14.9 \\
-11.2\end{array}$ & 1, AG Cnc \\
\hline $1234^{c}$ & 53 & 12.65 & 0.57 & $4.35563(25)$ & 0. & 1 & $\mathrm{n}$ & & & & & 2 \\
\hline 1237 & 52 & 10.78 & 0.94 & $697.8(7)$ & $0.105(15)$ & 5 & $\mathrm{n}$ & & & & & 2 \\
\hline 1242 & 50 & 12.72 & 0.68 & $31.7797(27)$ & $0.664(18)$ & 1,5 & $\mathrm{n}$ & & & & & 2 \\
\hline 1270 & 43 & 12.73 & 0.58 & & & 1,5 & $\mathrm{n}$ & & & & & \\
\hline 1282 & 40 & 13.33 & 0.56 & & & $1,4,5$ & $\mathrm{y}$ & $\begin{array}{l}0.360452(8)^{a} \\
0.360452(8)^{a}\end{array}$ & $\begin{array}{l}\text { [all; } B \text { ] } \\
\text { [all; } V \text { ] }\end{array}$ & $\begin{array}{l}0.05 \\
0.05\end{array}$ & $\begin{array}{l}-19.2 \\
-21.9\end{array}$ & AH Cnc \\
\hline
\end{tabular}

\footnotetext{
${ }^{a}$ Period does not correspond to the highest peak in the computed periodogram.

${ }^{b}$ Folded light curves do not look convincing.

${ }^{c}$ This system is a triple system; the period listed is for the inner binary.
}

interchanges minima. The period of 0.22072 days coincides with a nearby peak of similar height. $U$ data were only obtained during run 5 and cannot provide an equally precise period. In Fig. 3 the light curves are folded on the double period of 0.44144 days.

A light curve folded on the period given by Gilliland et al. shows that this period cannot be correct for the epoch of our observations. Since Gilliland et al. do not specify the error in the period, we cannot tell whether the period has changed significantly.

We see no significant changes in any of the colours $U-V, B-V$ or $V-I$.

\subsubsection{Spectroscopic binaries}

We note that the light curves of the spectroscopic binaries are given as function of orbital phase where phase 0 corresponds to the moment of maximum positive radial velocity of the primary star (primary receding).

S 999 The $B$ and $V$ data of the first run show variability with a semi-amplitude of $\sim 0.03 \mathrm{mag}$. Only the period found in the $B$ data has a false alarm probability smaller than $1 \%$. The highest peak in the periodogram is found at $4.6 \pm 1.0$ days (Fig. 2), but this period does not produce a smooth light curve. We suggest that the peak at 

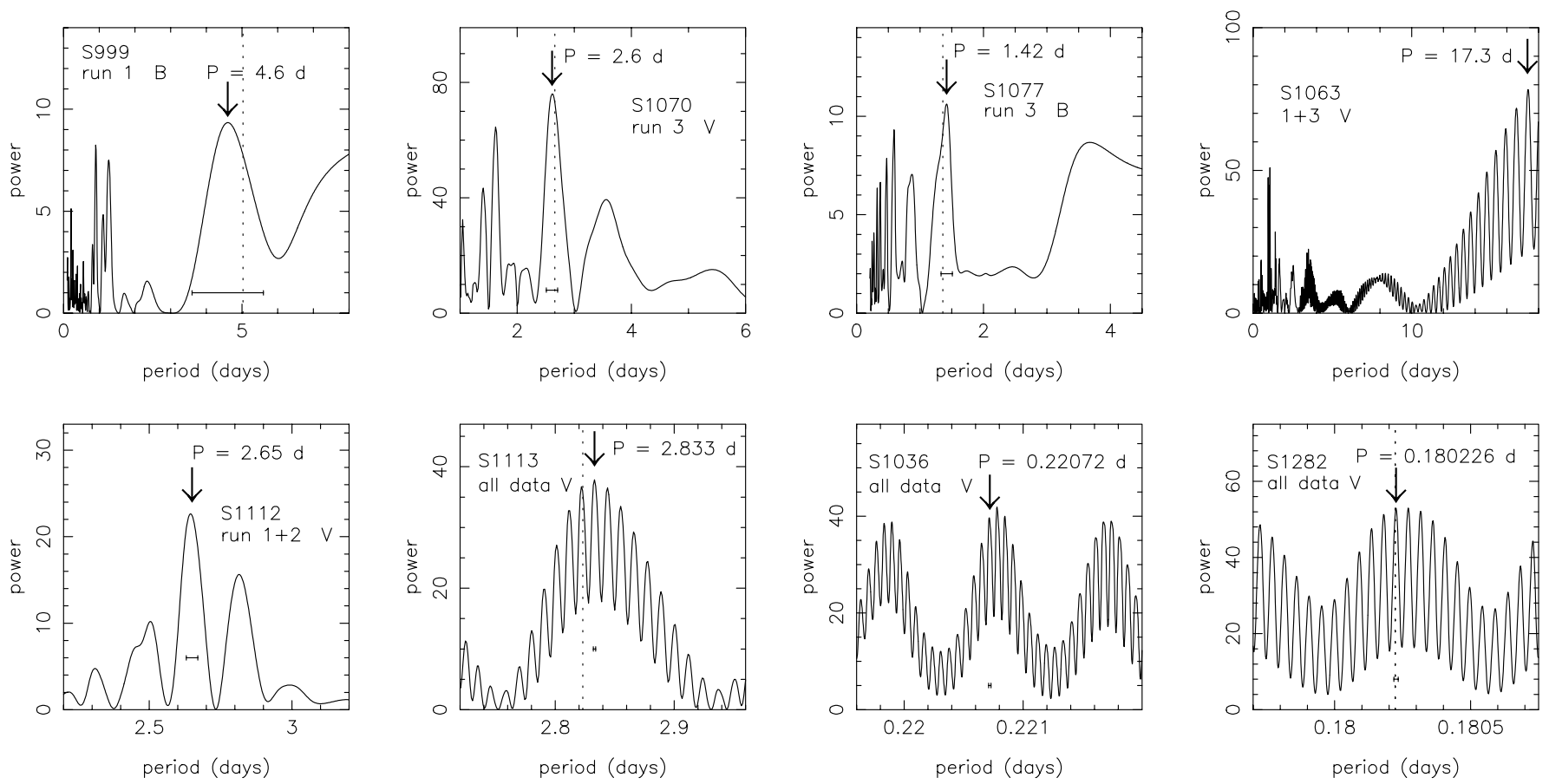

Fig. 2. Periodograms for the variable stars. The arrow indicates the photometric period or half the photometric period listed in Table 2. The dotted line marks the position of the orbital period for S 1070, S 1077 and S 1113, half the orbital period for S 999 and the position of the period as predicted by Kurochkin (1979) for S 1282. The horizontal errorbars give our estimate for the uncertainty in the period.

4.6 days is a harmonic of photometric variation on or near the orbital period of 10.06 days. Note that the duration of the first run was 10 nights, so our period search does not extend up to the orbital period. Gilliland et al. (1991) report a period of 9.79 days (no error is given) with an amplitude of only $0.013 \mathrm{mag}$.

In Fig. 4 we fold the $B$ and $V$ data on the orbital period. According to the orbital solution of Mathieu et al. (1990), the minimum brightness occurs around orbital phase 0 . The $B-V$ colour does not vary significantly.

S 1070 A period of $2.6 \pm 0.1$ days is detected in the $B$ and $V$ light curves of run 3 with a semi-amplitude of the variation of $\sim 0.03 \mathrm{mag}$.

The photometric properties of S 1070 have changed with respect to run 1 . If the variations seen in the third run $(\sigma \approx 0.014 \mathrm{mag}$ in $V)$ were present in the first run, they would have been detected.

In Fig. 4 all data of run 3 are folded on the orbital period which is compatible with the photometric period. The photometric minimum occurs around orbital phase 0.1-0.2 (ephemeris from Latham et al., private communication). The $V-$ Gunn $i$ colour curve shows periodic variations with a semi-amplitude of $\sim 0.03$ mag such that the star becomes bluer as it gets brighter.

S 1077 All light curves of this star are marked as variable except for the $U$ and $B$ data of run 5, probably due to the reduced sensitivity of run 5 , and the $V$ and $I$ data of run 1. The latter can point at a real absence of variation as could be the case in $\mathrm{S} 1070$; the variations of run $3(\sigma \approx 0.018 \mathrm{mag}$ in $V)$ were not seen in run 1 .
In the periodograms of the combined data peaks with a false alarm probability smaller than $1 \%$ are found near 0.6 and 1.3 days. However, when folded on these periods, the light curves do not look smooth. Therefore we also analyzed data of the different runs separately. Only the light curves of run 3 , with the highest precision, look smooth when folded on the periods of about 1.4 days (see Table 2) which have a false alarm probability smaller than $1 \%$ only in $B, V$ and Gunn $i$. This period does not correspond to the highest peak in the $V$ periodogram, which is found at 0.60 days. The semi-amplitude of the variation is small, $\sim 0.03$ in $V$.

The photometric period is compatible with the orbital period; we consider the latter to be the true period for the photometric variability. The data of run 3 are folded on the orbital period in Fig. 4. The photometric minimum occurs around phase $0.9-0$. The colours do not vary significantly.

S1113 As already noted by Kaluzny \& Radczynska (1991) this star is a photometric variable. In our observations, the $B$ and $V$ data cover the longest timespan and can therefore provide the most accurate photometric period. The periodogram is computed for 25000 periods to make the period bins smaller than the accuracy of our period determination (0.001). The periodogram (see Fig. 2) again shows fine structure with a spacing as expected from the two-year gap between runs 1 and 5. The maximum peak indicates a period of $2.833 \pm 0.001$ days, which is not compatible with the orbital period. The orbital period corresponds to a neighbouring peak in the periodogram at $2.822 \pm 0.001$ days (in $V ; 2.823 \pm 0.001$ days in $B$ ). Given 

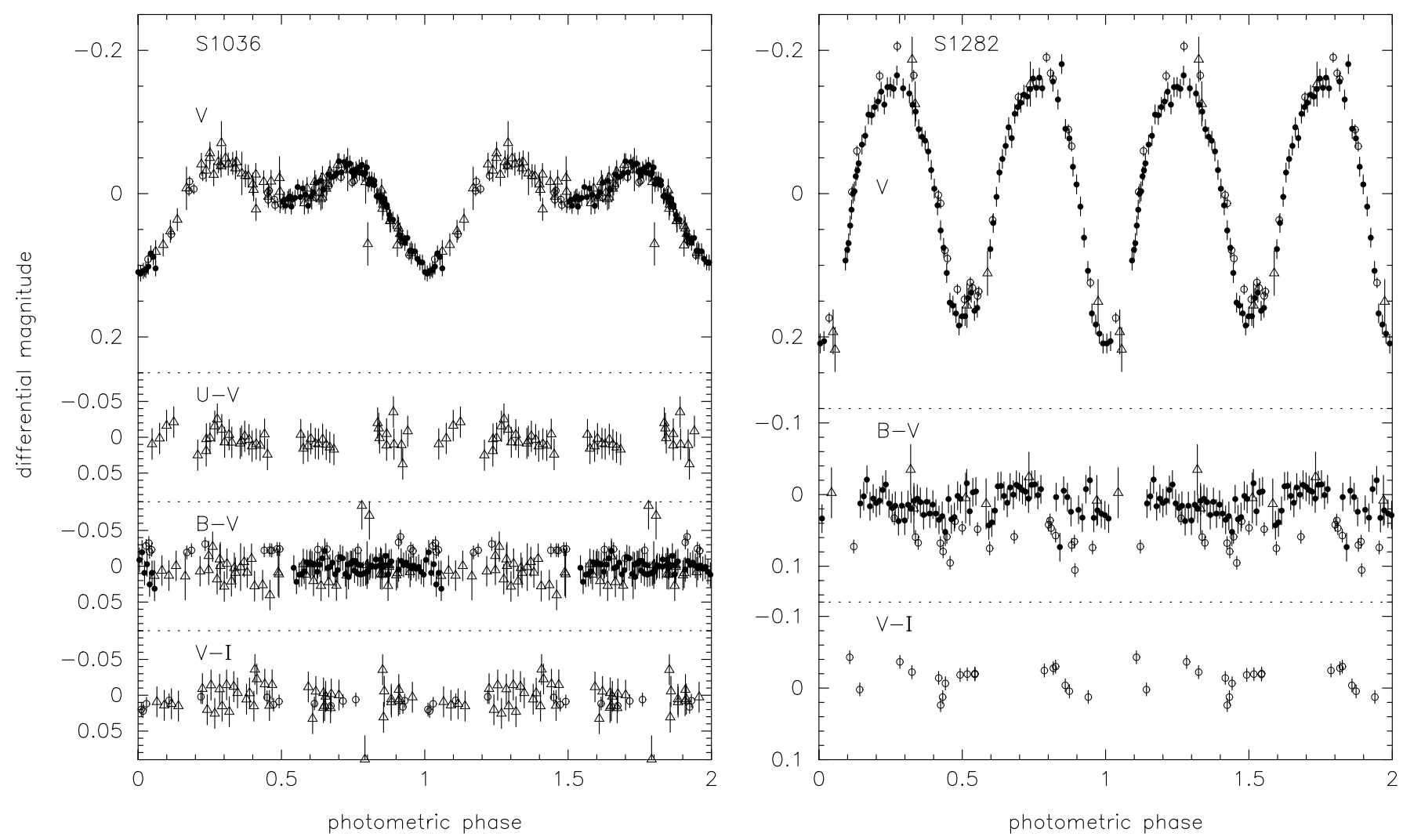

Fig. 3. Light curves of the two contact binaries S 1036 (EV Cnc) and S 1282 (AH Cnc) folded on the newly derived periods (see Table 2). Data from different observing runs are marked with different symbols: open circles for run 1, filled circles for run 4 and open triangles for run 5 .

the lack of significant difference in peak heights, the photometric and orbital periods may be the same. Therefore, the light curves in Fig. 4 are folded on the orbital period. For the $B$ light curve we find a similar result. The periods found in the $U$ and $I$ band are compatible, but less accurate (see Table 2).

Data taken during different runs at the same orbital phase give rise to scatter in the light curve, as seen in the top panel of Fig. 4. This could be explained if the amplitude, phase and/or the period of the variability has changed between the runs. When analyzed separately, the light curves of run 1 and 2 give a period of $2.8 \pm 0.1$ and $2.83 \pm 0.03$ days, respectively; the uncertainty is too large to detect a period change. Assuming that the photometric period is the orbital period we conclude that the amplitude or the phase of the variations has changed, either of which is possible if the variation is caused by a star spot.

We show separately the light and colour curves from runs 1 and 3 in the lower panels of Fig. 4. The colour variation is significant only in $V-I$ in run 1 and $B-V$ and $V$ - Gunn $i$ in run 3, such that the star becomes bluer as it brightens.

\subsection{3. $\mathrm{S} 1112$}

The data of the first $(B, V, I)$ and second $(V)$ runs show variability but only in the $B$ and $V$ light curves do we find significant periods of $2.7 \pm 0.2$ and $2.65 \pm 0.03$ days, respectively. In Fig. 4 the data are folded on the latter period. The amplitude of the variation is again small, only $\sim 0.04$ mag. Weak colour variations are only seen in $V$ - Gunn $i$ and appear to be in phase with the light curve; the star becomes bluer as it brightens. No information on binarity from radial-velocity measurements exists for this star.

\subsection{Non-periodic variable: $S 1063$}

Photometric variability of this star up to 0.18 mag was inferred by Racine (1971) from the differences between published values of the magnitude. Rajamohan et al. (1988) and Kaluzny \& Radczynska (1991) also noted its variability, but only the latter provide light curves (for Dec. 9 to 15 1986, see Fig. 5). This binary was included in all our runs except the second. The light curves of run 1 and 3 clearly show variability (see Fig. 5) on a long time scale. The variability during run 1 is similar to that observed by Kaluzny \& Radczynska. The longest interval of continuous observation was eighteen consecutive nights during run 3 , which is almost the length of the orbital period (18.39 days). Therefore during any one run we could not have established periodicity on the orbital period.

Data from run 1 and 3 were combined to look for periods up to 18 days. The highest peaks in the periodogram 

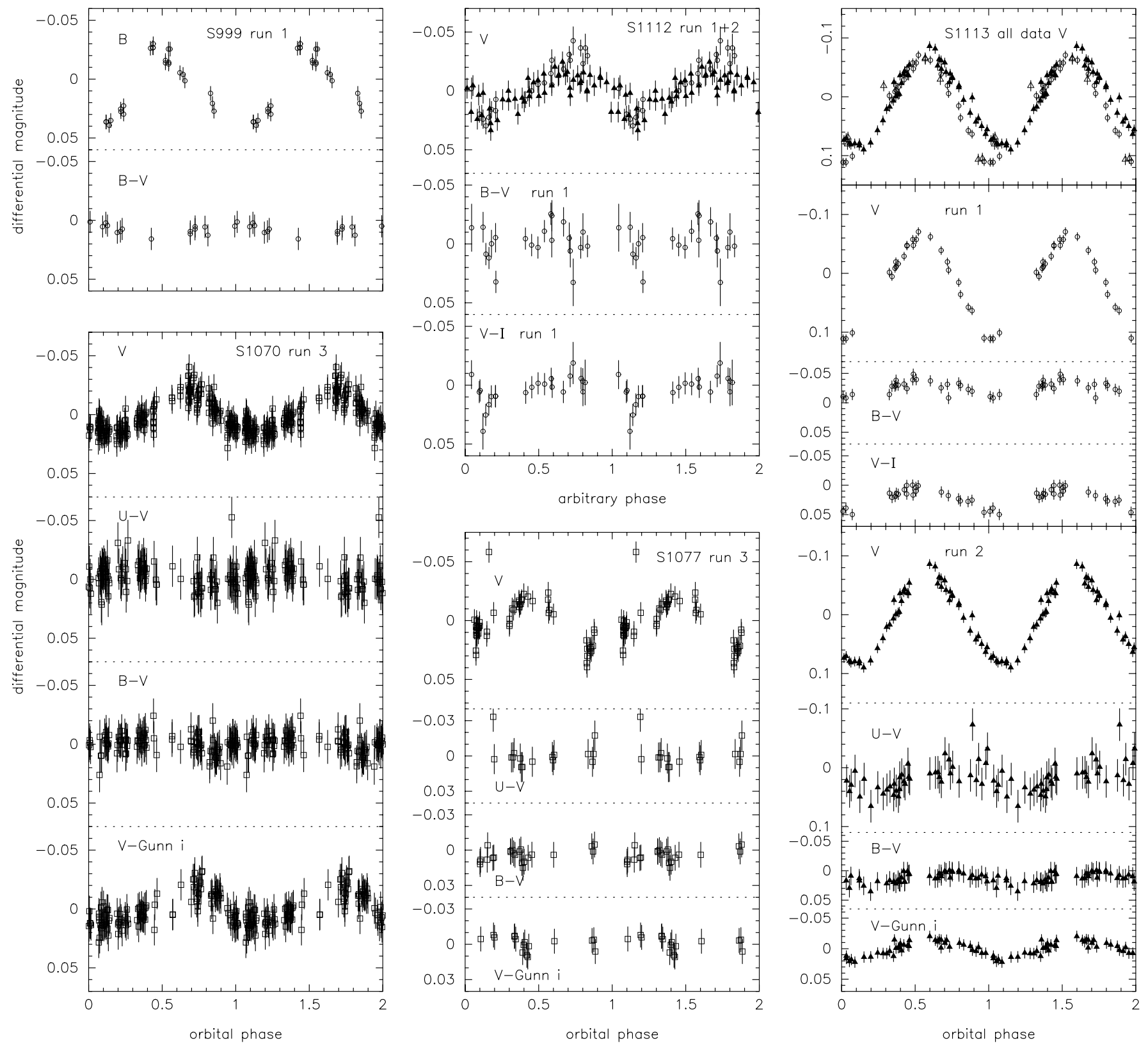

Fig. 4. Light curves of S 1112 (folded on the photometric period) and of S 999, S 1070, S 1077 and S 1113 (folded on the orbital periods; see Table 2). Data from different observing runs are marked with different symbols: open circles for run 1, filled triangles for run 2, open squares for run 3, filled circles for run 4 and open triangles for run 5 .

are found between 17 and 18 days, all with a false alarm probability smaller than $1 \%$. However, the folded light curves show no convincing periodicity. Therefore, no actual period is specified in Table 2 .

The amplitude of the variation increases towards the blue.

\section{Discussion}

We have studied the optical photometric properties of $\mathrm{X}$-ray sources in M67. Eight photometric variables, including three new variables, were found among the twenty two sources that are discussed in this paper. In all cases the amplitudes of the variations are small, ranging from 0.03 to 0.4 mag in $V$.

The light curves of the two contact binaries S 1036 and S 1282 arise through partial eclipses and ellipsoidal variations of the tidally deformed stars. Their X-rays are believed to be emitted by the hot coronae of the magnetically active components.

The primary and secondary eclipses of contact binaries usually are of similar depth. This has been interpreted as evidence that both stars have almost the same temperature, which in turn is evidence for energy exchange between the two stars in contact. Unequal depths of the 


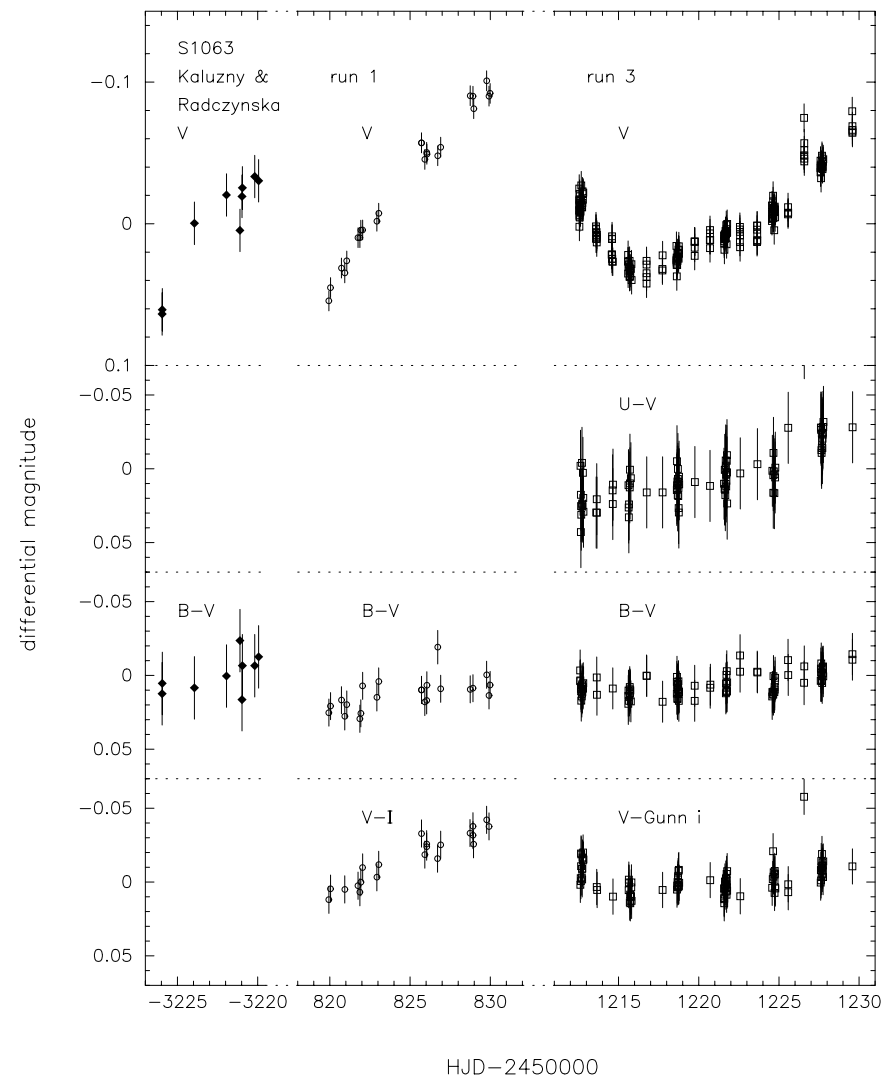

Fig. 5. Light curves and colour curves of S 1063. Data from different observing runs are marked as in Fig. 3. The data from Kaluzny \& Radczynska (1991) of Dec. 9-15 1986 are included on the left; the brightness variations are defined with respect to the mean magnitude of the lightcurve.

primary and secondary eclipses then implies different temperatures for both stars, i.e. poor thermal contact. The thermal contact can be suppressed when the system becomes (semi-)detached. It has been suggested that such phases of poor thermal contact occur periodically in contact binaries (Lucy \& Wilson 1979). In view of this interpretation it may appear surprising that we see unequal eclipses but no evidence of colour i.e. temperature variations in S 1036.

S 1036 is interesting as either an immediate progenitor of a contact binary, or because it is in the semi-detached phase of the thermal cycle of a contact binary. The upper limit on the colour variations in S1036 is about 0.05 in $B-V$ (Fig. 3). Radial-velocity measurements are required to determine the evolutionary status of this system and to convert the upper limit to the colour variations into an upper limit on temperature difference.

The small amplitude of the S 1036 light curve indicates either a small inclination or an extreme mass ratio (e.g. Rucinski 1997). In a volume-limited sample of contact binaries, Rucinski (1997) found that only two among the 98 systems have light curves with unequal minima. One of those two also has a relatively small amplitude of variation of about $0.25 \mathrm{mag}$.
Another feature of W UMa light curves associated with unequal eclipses is that at first quadrature (phase 0.25) the star is brighter than at second quadrature (phase 0.75). This has been explained with a hot spot on the secondary, possibly as a result of mass transfer in a semi-detached system (e.g. Rucinski 1997). This effect is also visible in S 1036 .

Within the two years of our observations we see evidence for variability of the light curve of S 1282: the secondary minimum of run 1 appears to be less deep and flatter than observed in run 4. A similar variation was seen by Gilliland et al. (1991) who noted that in their observations of 1988 the secondary eclipse had a flat shape, while the observations by Whelan et al. (1979) done from 1973 to 1976 showed a rounded secondary minimum. The timescale of these variations is indicative of the presence of spots.

The light curves of the periodic variables S 999, S 1070, S 1077 and S 1113 display only one maximum per cycle. S 1113 and S 1070 also show colour variations in phase with the brightness. For all four systems there is indication for variability in the light curves. The amplitude of the variation in S 999 is different in our observations and those of Gilliland et al. (1991); in S 1070 and S 1077 there has likely been a change between run 1 and run 3 and in S 1113 between run 1 and 2 . The short timescale of this variation is an indication of brightness modulations by spots. Remarkably, in all cases the photometric minimum occurs around orbital phase 0 . We have no explanation for this.

All four systems are spectroscopic binaries with photometric periods compatible with the orbital period. The circular orbital periods, their X-ray luminosity, and the CaII K emission in the case of S 999, S 1077 and S 1113 (Pasquini \& Belloni 1998; van den Berg et al. 1999) make these stars likely candidates for magnetically active systems due to one or both stars being tidally locked. This was already suggested by Belloni et al. (1998) and our light curves support their interpretation.

The light curve of $\mathrm{S} 1112$ shows low-amplitude periodic light and colour variations similar to those seen in these four binaries. This star has not been monitored for radialvelocity variations but the X-ray luminosity and the light curve are typical for magnetically active systems which suggests that S1112 is a binary with an orbital period of about 2.65 days.

We do not understand the variability that we observe for S 1063. The source shows spectroscopic signatures of magnetic activity (van den Berg et al. 1999). However, if one of the stars in this binary would be corotating near periastron, we expect a rotation period of 14.6 days (Eq. (42) of Hut 1981) which is excluded by the observations of run 3. We conclude that either the star does not vary periodically or that the period of variability is longer than 18 days. More observations covering a longer timespan are required to understand the nature of the variability. Our findings are in contrast with the suggestion by 
Kaluzny \& Radczynska (1991) that S 1063, as well as S 1113, are highly evolved W UMa-type binaries.

\section{Conclusion}

Of the twenty two X-ray sources in M67 that we discuss, sixteen are spectroscopic binaries with known orbital periods. Our survey for optical photometric variables among these X-ray sources has established eight variables. Seven of these are among the sixteen binaries, the binary status of the eighth, S1112, is not yet known. In addition, Gilliland et al. (1991) observed periodic optical variation in three more of the X-ray binaries with amplitudes too low to be detected by us: S 1019 (semi-amplitude $0.015 \mathrm{mag}$ ), S1242 (semi-amplitude $0.0025 \mathrm{mag}$ ) and S 1040 (semi-amplitude 0.012) mag. Thus ten of the sixteen X-ray binaries in M67 are optical variables at the $\gtrsim 0.01 \mathrm{mag}$ level.

Belloni et al. (1998) have suggested that rapid stellar rotation resulting from tidal locking results in enhanced magnetic activity and X-ray emission. Figure 6 shows the visual magnitude versus orbital period of the spectroscopic binaries. With the exception of S 1040 and S 1112, all variables have orbital periods less than 20 days and $V>15$. In all cases but S 1019 and S 1063, the photometric period is equal to the orbital period or, in the case of S 1242, the orbital period near periastron. Evidently tidal locking has been established, leading to rotation of at least the primary star that is more rapid than typical for solar-mass stars at 4 Gyr. Thus our results establish a key premise of the Belloni et al. (1998) picture for the X-ray emission. Furthermore, if the cause of the observed optical variability is indeed spot modulation of the observed flux, then the presence of the required large spots is consistent with enhanced magnetic activity in these stars. The X-ray emission and optical variability properties of S 1019 and S 1063 require further investigation.

Three binaries were not detected as variables despite their short orbital periods. S 972 is the faintest of the binary sample at $V=15.37$, and so its variability may have gone undetected. The X-ray luminosities of S 1045 $\left(P_{\text {orb }}=7.6\right.$ days $)$ and $\mathrm{S} 1234\left(P_{\text {orb }}=4.3\right.$ days $)$ are among the lowest of the binary X-ray sources and indicate low activity levels; this can explain the absence of optical variability due to spots. Rajamohan et al. (1988) have noted S 1234 as a possible optical variable (semi-amplitude $\sim 0.16 \mathrm{mag}$ ) which suggests that time-variability of the spot phenomenon can also explain the absence of optical variation.

The interpretation of S 1040 and of the remaining three X-ray binaries S 760, S 1072 and S 1237 may be the most challenging. All have long orbital periods. Given their wider separations tidal locking is not expected and so the consequent stellar rotations may be characteristic of single stars. As such, their lack of large spots and consequent photometric variability is not a surprise. Nonetheless, these binaries are X-ray sources. Their X-ray emission remains to be explained.

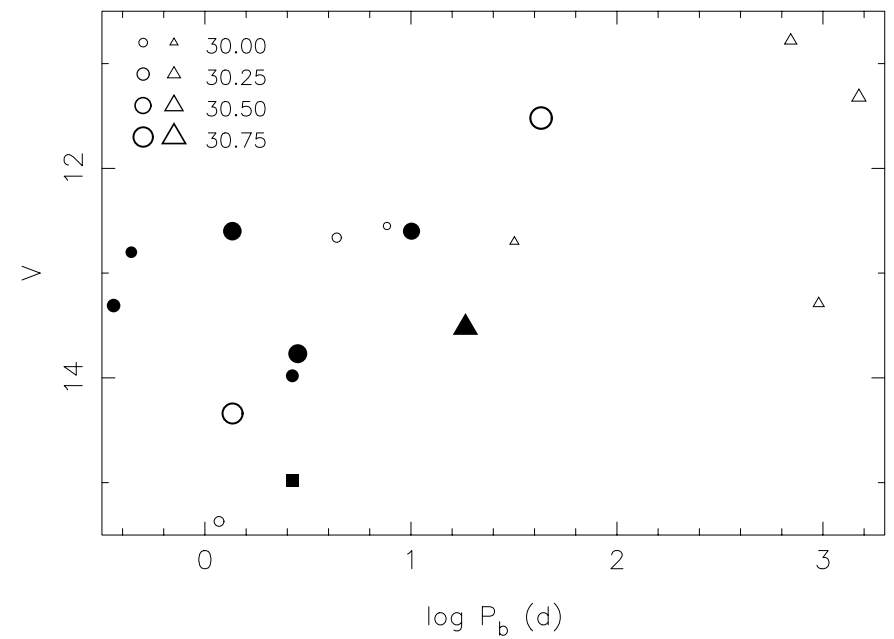

Fig. 6. Visual magnitude versus orbital period of the M67 binaries detected in X-rays. The size of the symbol is a measure for the logarithm of the X-ray luminosity $(0.1-2.4 \mathrm{keV}$, in $\mathrm{erg} \mathrm{s}^{-1}$ ) as indicated in the figure. Eccentric binaries are indicated with triangles, binaries with eccentricities compatible with zero (within the $3 \sigma$ error) with circles. Filled symbols are systems for which we detected photometric variability. S 1112 is indicated with a filled square.

No large radial-velocity variations were found for S 775 and $\mathrm{S} 1270$ ( $\sigma$ is $0.9 \mathrm{~km} \mathrm{~s}^{-1}$ in 12 observations spanning 5200 days and $0.7 \mathrm{~km} \mathrm{~s}^{-1}$ in 7 observations spanning 800 days, respectively, see Mathieu et al. (1986)); if these stars are binaries their periods must be relatively long. Thus their X-ray luminosities, as those of S 364, S 628 and S 1027 for which no radial-velocity information is available, remain unexplained.

Acknowledgements. The authors wish to thank Magiel Janson, Rien Dijkstra, Gertie Geertsema, Remon Cornelisse and Gijs Nelemans for obtaining part of the data used in the paper. The authors want to thank David Latham for computing preliminary orbital solutions for four spectroscopic binaries to support this research; the radial-velocity measurements are part of a larger study of M 67 binaries carried out by D. W. Latham, A. A. E. Milone and R. D. Mathieu. The Kitt Peak National Observatory is part of the National Optical Astronomy Observatories, which is operated by the Association of Universities for Research in Astronomy, Inc. (AURA) under cooperative agreement with the National Science Foundation. The Jacobus Kapteyn Telescope is operated on the island of La Palma by the Isaac Newton Group in the Spanish Observatorio del Roque de los Muchachos of the Instituto de Astrofisica de Canarias. The Dutch $0.91 \mathrm{~m}$ Telescope was operated at La Silla by the European Southern Observatory. MvdB was supported by the Netherlands Organization for Scientific Research (NWO).

\section{References}

Belloni, T., Verbunt, F., \& Mathieu, R. D. 1998, A\&A, 339, 431 
Gilliland, R. L., Brown, T. M., Duncan, D. K., et al. 1991, AJ, 101,541

Honeycutt, R. K. 1992, PASP, 104, 435

Horne, J. H., \& Baliunas, S. L. 1986, ApJ, 302, 757

Hut, P. 1981, A\&A, 99, 126

Kaluzny, J., \& Radczynska, J. 1991, IBVS, 3586

Kurochkin, N. E. 1960, Astron. Circ. USSR, 212, 9

Kurochkin, N. E. 1979, Astron. Circ. USSR, 1076, 2

Lucy, L. B., \& Wilson, R. E. 1979, ApJ, 231, 502

Mathieu, R. D., Latham, D. W., \& Griffin, R. F. 1990, AJ, 100, 1859

Mathieu, R. D., Latham, D. W., Griffin, R. F., \& Gunn, J. E. 1986, AJ, 92, 1100

Montgomery, K. A., Marschall, L. A., \& Janes, K. A. 1993, AJ, 106,181

Pasquini, L., \& Belloni, T. 1998, A\&A, 336, 902
Pols, O. R., Schröder, K. -P., Hurley, J. R., Tout, C. A., \& Eggleton, P. P. 1998, MNRAS, 298, 525

Racine, R. 1971, ApJ, 168, 393

Rajamohan, R., Bhattacharyya, J. C., Subramanian, V., \& Kuppuswamy, K. 1988, BASI, 16, 139

Rucinski, S. M. 1997, AJ, 113, 1112

Sanders, W. L. 1977, A\&AS, 27, 89

Scargle, J. D. 1982, ApJ, 263, 835

Stassun, K. G., van den Berg, M., Mathieu, R. D., \& Verbunt, F. 2002, A\&A, 382, 899, Paper II

van den Berg, M., Orosz, J., Verbunt, F., \& Stassun, K. G. 2001, A\&A, 375, 375

van den Berg, M., Verbunt, F., \& Mathieu, R. D. 1999, A\&A, 347,866

Whelan, J. A. J., Worden, S. P., Ruciński, S. M., \& Romanishin, W. 1979, MNRAS, 186, 729 